\title{
Double fingerprint characterization of uracil and 5-fluorouracil
}

\author{
Martin Perez-Estebanez, William Cheuquepan, Jose V. Cuevas-Vicario, Sheila Hernandez, \\ Aranzazu Heras, Alvaro Colina* \\ Department of Chemistry, Universidad de Burgos, Pza. Misael Bañuelos s/n, E-09001 Burgos (Spain)
}

\section{A R T I C L E I N F O}

\section{Article history:}

Received 5 March 2021

Revised 16 April 2021

Accepted 8 May 2021

Available online 20 May 2021

\section{Keywords:}

Spectroelectrochemistry

Raman

EC-SERS

EC-SOERS

\begin{abstract}
A B S T R A C T
Time Resolved Raman spectroelectrochemistry (TR-Raman-SEC) has been used for the first time to obtain two different Raman spectra of one single analyte in the same experiment. This double detection has been accomplished thanks to the use of electrochemical surface enhanced Raman scattering (EC-SERS) and electrochemical surface oxidation enhanced Raman scattering (EC-SOERS) in the same experiment. These two Raman enhancement phenomena can provide a broad insight into the interaction between analyte and substrate surface when they are combined. To prove the possibilities of this methodology, a Raman spectroelectrochemistry study of uracil (U) and 5-fluorouracil (5-FU), two analytes with relevance in medicine and biochemistry, have been performed. Density functional theory (DFT) calculations has been carried out to shed more light on the interaction of these molecules with silver substrates in acidic media.
\end{abstract}

(c) 2021 The Authors. Published by Elsevier Ltd. This is an open access article under the CC BY license (http://creativecommons.org/licenses/by/4.0/)

\section{Introduction}

Raman spectroscopy is a vibrational spectroscopy used for many years in the characterization of a wide range of molecules both in solid and in solution, mainly due to the capability of this technique to provide spectroscopic fingerprints of substances [1]. The low sensitivity of Raman spectroscopy limits its use both in the characterization and in the detection of molecules. Luckily, Raman signal can be enhanced using diverse strategies like resonance Raman, surface enhanced Raman scattering (SERS) or electrochemical surface oxidation Enhanced Raman scattering (EC-SOERS). The most used method to enhance the Raman signal is SERS. Particularly, electrochemical surface enhanced Raman scattering (EC-SERS) has been studied for the last 40 years [2]. It is well established that the observed Raman enhancement can be attributed to two main factors, namely electromagnetic enhancement (EM) and chemical enhancement (CE). The first one is related to the intensification of the electric field on the surrounding of a plasmonic nanostructure during localized surface plasmon resonance, and can be responsible of an enhancement factor of Raman scattering up to $10^{9}[3,4]$. On the other hand, the CE is related with the change of the polarizability of molecules due to charge transferences between the analyte and the substrate. The latter mechanism is related with lower enhancement factors than the EM, between $10^{2}$ and $10^{3}[4,5]$.

\footnotetext{
* Corresponding author.

E-mail address: acolina@ubu.es (A. Colina).
}

EC-SOERS is a recently discovered phenomenon that allows the Raman signal enhancement of molecules during the oxidation of a silver substrate [6]. This phenomenon is truly interesting, since its origin defies the classic theory of SERS, which suggest that Raman enhancement should disappear at anodic potentials due to the degradation of plasmonic properties $[7,8]$. Although the origin of EC-SOERS is still under discussion, it is clear that it provides a great reproducibility and selectivity in the enhancement of Raman signal of several analytes, which makes it a great method for the development of both qualitative and quantitative analytical methods [9-11].

Time resolved Raman spectroelectrochemistry (TR-Raman-SEC) is an instrumental technique in which Raman spectroscopy is used to interrogate the electrode surface during an electrochemical experiment. This combination allows us the simultaneous generation and characterization of a SERS substrate, tuning concomitantly the molecular resonances to the excitation wavelength, which provides information about the electronic states involved in SERS [4]. TRRaman-SEC is also a great method to resolve complex mixtures, where strong spectroscopic interferences are present [10].

To extract all the information contained in Raman spectra, a proper assignation of Raman bands is required. This assignation has been usually performed by comparison between Raman and IR spectra, or by DFT calculations [12-14]. Nevertheless, this assignation is not always easy, since discordances between calculated and experimental spectra can be significant. This aspect is especially difficult when phenomena like SERS or EC-SERS are used to 
enhance the experimental signal, since these phenomena involve the interaction of molecules with metallic structures and the influence of electric fields, which might not be easy to compute [15].

In this work, we present a new experimental methodology based on TR-Raman-SEC that allows the acquisition of two different Raman spectroscopic signals of one analyte in a single experiment, thanks to the presence of two different Raman enhancement phenomena: EC-SERS and EC-SOERS. These phenomena provide two different but characteristic spectroscopic fingerprints of analytes. Therefore, we can perform a double detection of several analytes with these two signals in a single TR-Raman-SEC experiment.

To demonstrate the possibilities of the double spectroscopic fingerprint provided by our methodology, we performed the detection of uracil (U) and 5-fluorouracil (5-FU), two analytes with relatively simple structure but with great relevance in biochemistry.

$\mathrm{U}$ is a molecule present in several biological processes; from the formation of RNA to metabolic routes in some bacteria $[16,17]$. It is also involved in the generation of several DNA mutations, [18]. $U$ is a key molecule in biology, and its derivatives have several applications in pharmacology. A great example is 5-FU, an antimetabolite drug widely used in the treatment of colorectal and breast cancer, among others, for the last 40 years [19-21]. This drug is rapidly included into the metabolism, where it inhibits the activity of the thymidylate synthase, hindering the conversion of uridine monophosphate into thymidine monophosphate and negatively affecting DNA replication $[19,20]$. However, the usage of 5-FU has some drawbacks, since it has been observed that 5-FU can be easily incorporated to RNA, disrupting its normal processing $[19,22,23]$. 5-FU has been also identified as a source of DNA mutations that might increase tumor evolution after treatment [20]. For these reasons, a great interest exists on the development of facile, sensitive and selective analytical methods to detect and quantify both, 5-FU and U.

Traditionally, 5-FU and $U$ analysis has been performed with techniques such as liquid chromatography-mass spectrometry/mass spectrometry (LC-MS/MS) [24], electrochemistry [25,26] and fluorescence $[27,28]$. Some of them require a complex sample pretreatment and others can be sensitive to the presence of interferences.

SERS, however, has managed to overcome these problems. For example, it has been used for in vivo measurements in tumor detection with very simple sample pretreatment [29,30]. In genomic analysis, SERS has been able to detect specific targeted DNA strands [31] or even specific genomic mutations in a single strand $[32,33]$. Coupling of this technique with multivariate analysis has demonstrated to be a powerful tool to study complex systems, giving, for example, important information about the structure of nucleobases and their interactions [30,34].

In this work, we present a new methodology that allows the double detection of $U$ and 5-FU, thanks to the EC-SERS and ECSOERS phenomena. This method can be used for a more reliable analytical detection as well as providing more spectroscopic information of the structures involved in the electrode transformation, giving more data for possible computational calculations of spectroelectrochemical systems.

\section{Methods and materials}

\subsection{Reagents and solutions}

Perchloric acid $\left(\mathrm{HClO}_{4}, 60 \%\right.$, Sigma-Aldrich), potassium chloride (KCl, 99\%, ACROS Organics), uracil (U, >99\%, Sigma-Aldrich), 5fluorouracil (5-FU, >99\% HPLC, Sigma-Aldrich). Reagents were used as received, without further purification. All solutions were prepared using ultrapure water obtained from a Millipore DirectQ pu- rification system provided by Millipore $(18.2 \mathrm{M} \Omega \cdot \mathrm{cm}$ resistivity at $\left.25^{\circ} \mathrm{C}\right)$.

\subsection{Instrumentation}

\subsubsection{Time resolved Raman spectroelectrochemistry}

In-situ TR-Raman-SEC was performed with a customized SPELEC-RAMAN instrument (Metrohm-DropSens), which included a $785 \mathrm{~nm}$ laser source. The laser power was set at $102 \mathrm{~mW}(325 \mathrm{~W}$ $\mathrm{cm}^{-2}$ ) in all experiments. This instrument was connected to a Raman probe (DRP-RAMANPROBE, Metrohm-DropSens). A homemade cell for screen-printed electrodes (SPEs) was used during the experiments. DropView SPELEC software (Metrohm-DropSens) was used to control the instrument, which allowed getting real-time and synchronized spectroelectrochemical data. An integration time of $1 \mathrm{~s}$ was used to obtain each Raman spectrum in all the TRRaman-SEC experiments. Spectral resolution was $<4 \mathrm{~cm}^{-1}$. Silver SPEs (Ag-SPE) from Metrohm DropSens (DRP-C013) were used as electrochemical set-up. These electrodes consisted of a working electrode of silver with a diameter of $1.6 \mathrm{~mm}$, a counter electrode of carbon and a pseudo-reference of silver.

Cyclic voltammetry was used as electrochemical technique to perform TR-Raman-SEC experiments. All potentials are referred to the pseudo-reference electrode of silver ( $v s$. Ag). Two voltammetric cycles were performed between $-0.40 \mathrm{~V}$ and $+0.40 \mathrm{~V}$, starting at $0.00 \mathrm{~V}$ in anodic direction for each experiment. A scan rate of $0.02 \mathrm{~V} \mathrm{~s}^{-1}$ and a step potential of $2 \mathrm{mV}$ were set for all electrochemical measurements. All solutions were prepared in $0.1 \mathrm{M}$ $\mathrm{HClO}_{4}$ and $10^{-2} \mathrm{M} \mathrm{KCl}$, with a concentration of analyte of $10^{-3} \mathrm{M}$. These experimental conditions were carefully chosen after a proper optimization process where $\mathrm{KCl}$ concentration, $\mathrm{pH}$ and potential window were evaluated. All experiments were performed thrice.

\subsubsection{Computational methods}

All theoretical calculations were performed using the functional PBE0 [35] including dispersion correction with the BeckeJohnson damping scheme (D3BJ) [36,37]. For the description of the atoms, basis def2-SVP [38] were used for non-metallic atoms and LANL2DZ [39] for silver atoms level of theory. The optimization was performed freezing the silver atoms, but without any restriction to the other atoms. The software package ORCA 4.2.1 has been used for these DFT calculations $[40,41]$.

\section{Results and discussion}

\subsection{Double fingerprint of one analyte in a single experiment}

TR-Raman-SEC allowed us to obtain the evolution of the Raman spectra on a Ag-SPE surface during a cyclic voltammetry experiment of a $10^{-3} \mathrm{M} \mathrm{U}$ solution in $0.1 \mathrm{M} \mathrm{HClO}_{4}$ and $10^{-2} \mathrm{M} \mathrm{KCl}$. Two different Raman enhancement phenomena can be observed, Fig. 1, during the experiment. Fig. 1A shows the cyclic voltammogram (CV) obtained during this spectroelectrochemistry experiment. When the applied potential reaches $+0.10 \mathrm{~V}$, the formation of $\mathrm{AgCl}$ is observed [6]. Next, at $+0.35 \mathrm{~V}$, the massive oxidation of the silver electrode, from $\mathrm{Ag}^{0}$ to $\mathrm{Ag}^{+}$, takes place. At this point, a notorious enhancement of the characteristic Raman signal of $U$ $\left(801 \mathrm{~cm}^{-1}\right)$ is observed due to the EC-SOERS effect $[6,10,11,42,43]$ (orange line, Fig. 1B). This enhancement reaches its maximum during cathodic scan, around $+0.30 \mathrm{~V}$. At this point, the reduction of $\mathrm{Ag}^{+}$takes place, and the EC-SOERS effect starts to decrease. Further, in the cathodic scan, $\mathrm{AgCl}$ deposited on the surface is reduced to $\mathrm{Ag}^{0}$ around $-0.10 \mathrm{~V}$. During this reduction, plasmonic silver nanostructures are produced on the electrode surface $[43,44]$. These nanostructures generate a second Raman enhancement of another Raman band of $U\left(768 \mathrm{~cm}^{-1}\right)$ due to EC-SERS effect (blue 

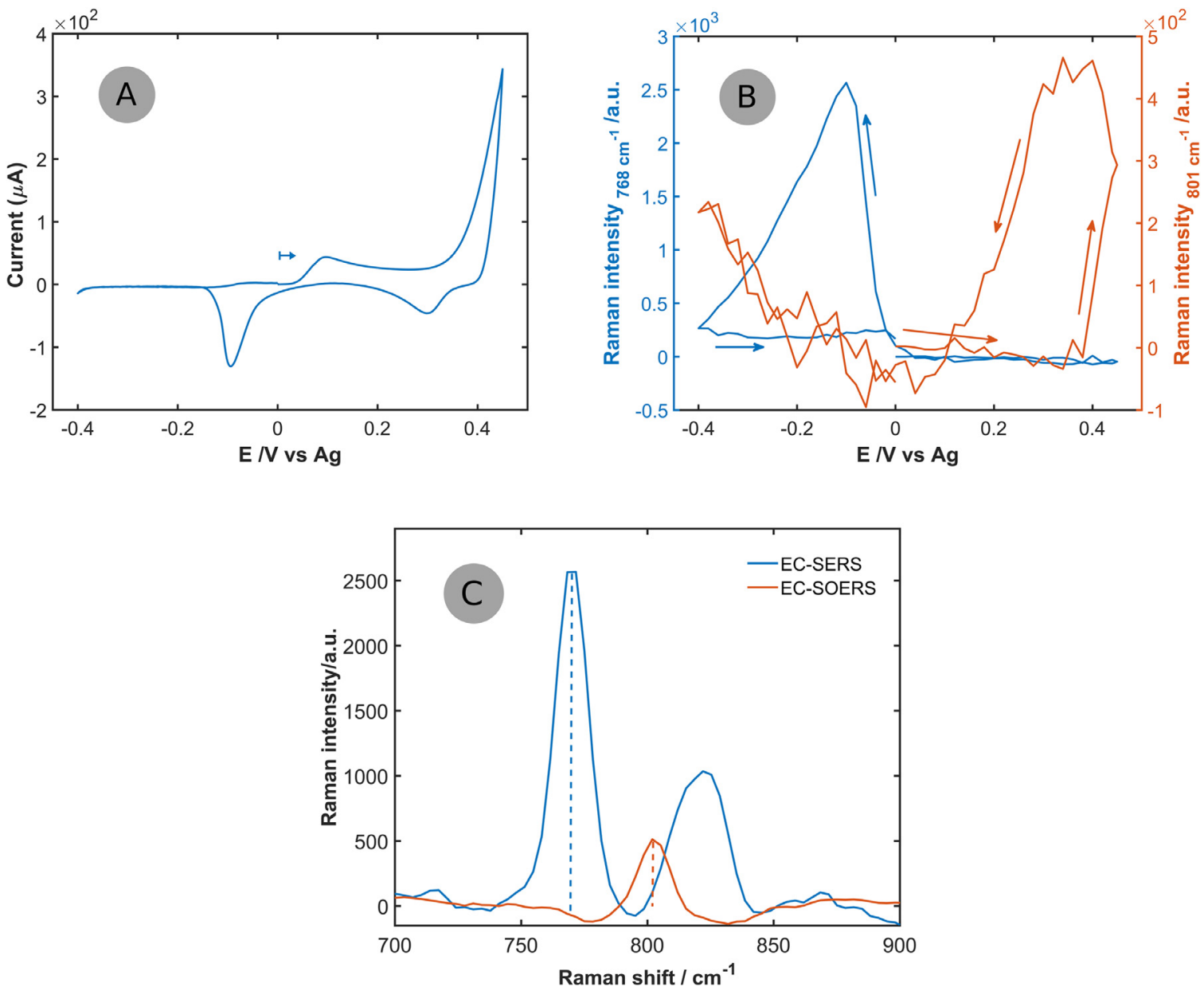

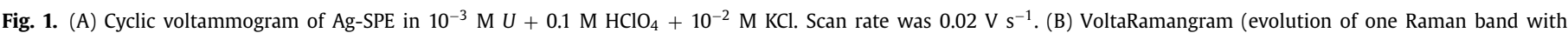

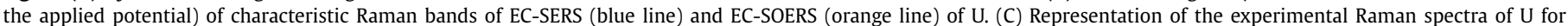

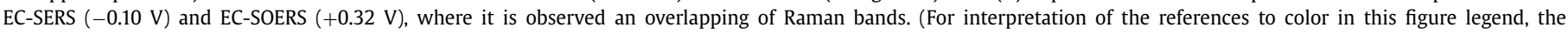
reader is referred to the web version of this article.)

line, Fig. 1B). This effect reaches its maximum around $-0.10 \mathrm{~V}$ and then decreases to a lower but stable signal. This behavior could be related with different processes: (1) the continuous modification of the nanostructures deposited on the electrode surface during $\mathrm{AgCl}$ reduction could lead to a loss of plasmonic properties, (2) the desorption of the analyte, or (3) a change in the orientation of the molecule during the adsorption.

An important detail should be denoted in Fig. 1B. In this figure, two different Raman shifts are plotted depending on the potential window where the Raman enhancements were observed. Spectra shown in Fig. $1 C$ depicts the Raman spectra of $U$ where the signal due to EC-SOERS and EC-SERS phenomena is maximum. The Raman spectrum observed in EC-SOERS (orange line, Fig. 1C) has some differences in the band Raman shifts with respect to the one observed in EC-SERS (blue line, Fig. 1C). Besides, it can be observed that the band at $801 \mathrm{~cm}^{-1}$ for EC-SOERS and the band at $822 \mathrm{~cm}^{-1}$ for EC-SERS are partially overlapped. The shifting of the Raman bands can lead to some misunderstandings during the interpretation of the evolution of Raman bands with the potential along the experiment (voltaRamangrams). For example, Fig. 1B shows that Raman intensity at $801 \mathrm{~cm}^{-1}$ is enhanced at potentials greater than $+0.20 \mathrm{~V}$, due to EC-SOERS, but also at potentials below $0.00 \mathrm{~V}$, which might lead us to think that this Raman signal is enhanced twice because of EC-SOERS and EC-SERS phenomena. However, from spectra in Fig. $1 C$ it must be concluded that this behavior is due to an overlapping of the bands linked to these phenomena. Therefore, we can deduce that two different spectra linked to the same molecule can be obtained, being related to the two different Raman enhancement phenomena.

Similar conclusions can be extracted from the study of 5-FU. Fig. S1, in supplementary materials, shows the CV and voltaRamangrams at two different Raman shifts during the TR-Raman-SEC experiment. The CV (Fig. S1A) is almost identical to the one observed for $U$ (Fig. 1A). As was described for $U$, it can be seen that some Raman shifts presents EC-SOERS effect (for example, $792 \mathrm{~cm}^{-1}$ ), since the enhancement of the Raman signal at anodic potentials is observed (orange line, Fig. S1B). Meanwhile, another Raman bands are enhanced at cathodic potentials due to EC-SERS effect (for example $764 \mathrm{~cm}^{-1}$, blue line, Fig. S1B). It can be also observed that the evolution of 5-FU Raman spectra during the experiment shows some overlapping of bands ascribed to EC-SOERS and EC-SERS phenomena. It can generate some misunderstanding on the interpretation of Raman signals evolution. In this case, the signal at 792 $\mathrm{cm}^{-1}$ seems to have negative Raman intensity at negative potentials. This is due to the difficulty associated to the generation of a good baseline that fits properly to all the Raman spectra obtained during the experiment. Fig. S2 shows the Raman spectra corresponding to the maximum EC-SOERS and EC-SERS enhancement of 5-FU. The Raman signal at $792 \mathrm{~cm}^{-1}$, which shows EC-SOERS enhancement, corresponds to a valley in the EC-SERS spectra. This generates an apparently anomalous behavior in the evolution of this Raman band, shown in Fig. S1B (orange line). 

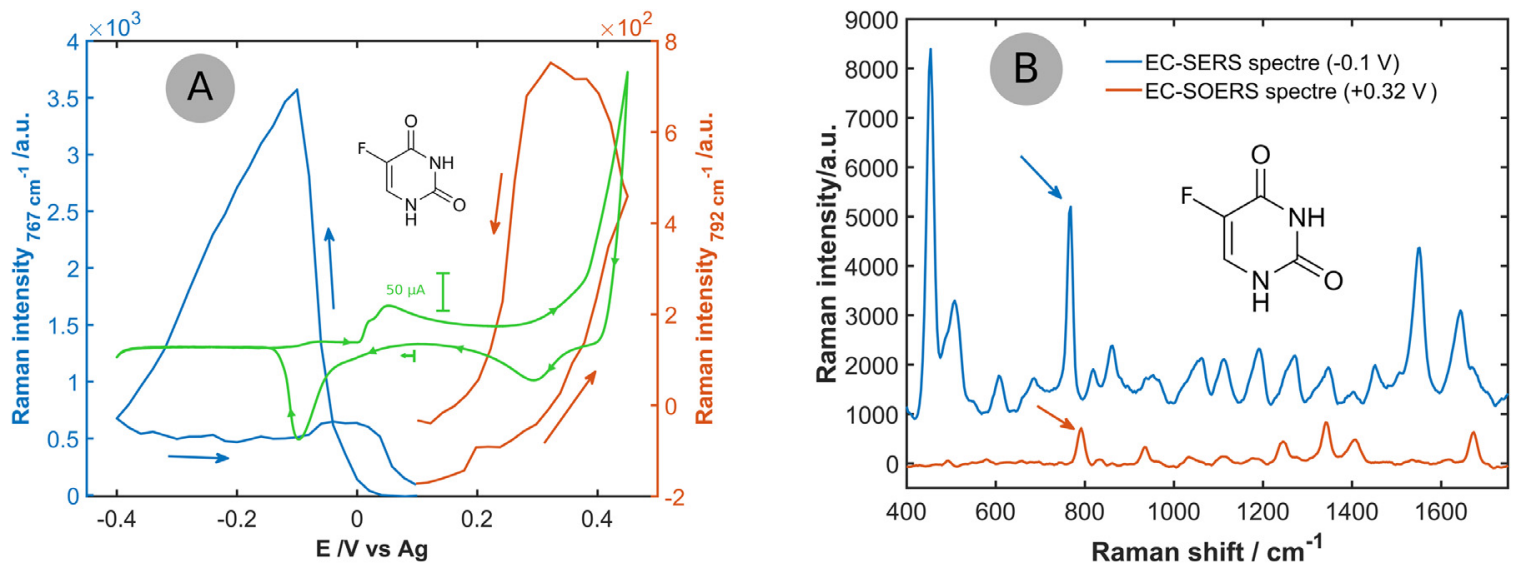

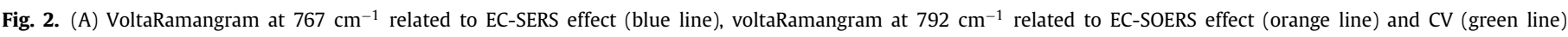

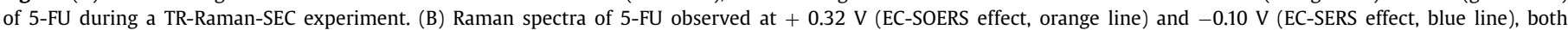

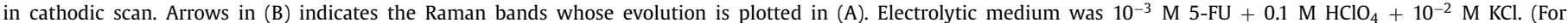
interpretation of the references to color in this figure legend, the reader is referred to the web version of this article.)
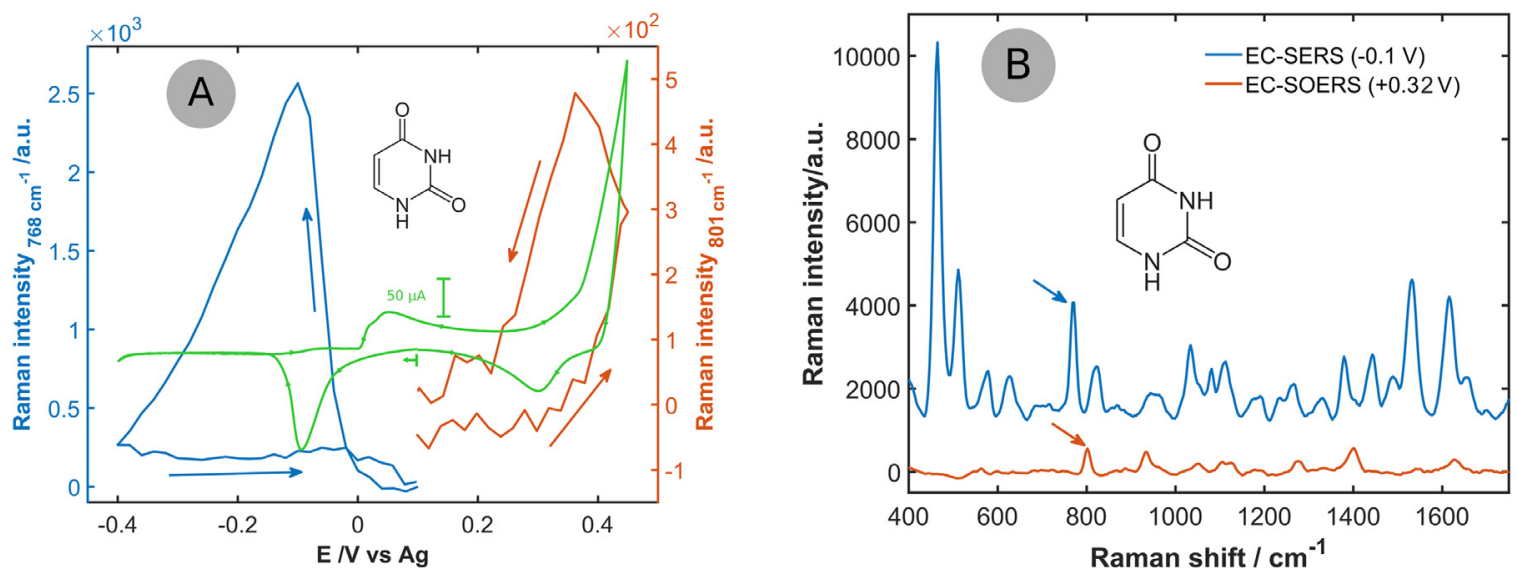

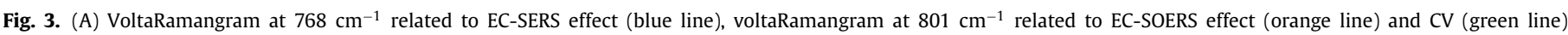

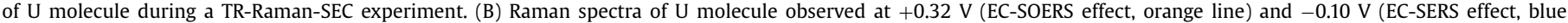

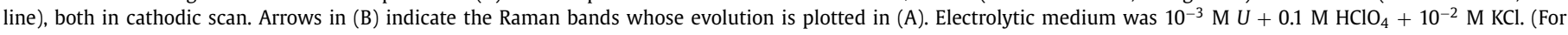
interpretation of the references to color in this figure legend, the reader is referred to the web version of this article.)

Until now, we have analyzed the evolution of Raman signals of $\mathrm{U}$ and 5-FU during the first voltammetric cycle applied to the electrode. However, the behavior of EC-SOERS and EC-SERS responses vary when several CVs are performed, as is shown in Figs. S3 and S4. In these figures, the voltaRamangrams for both EC-SOERS and EC-SERS phenomena of $U$ and 5-FU are presented along two consecutive CVs. U molecule seems to give similar Raman enhancements, no matter the scan (Fig. S3A and S3B), even though important differences are observed in the CVs (Fig. S3C). However, 5-FU molecule responses change significantly when two consecutive potential scans were carried out. VoltaRamangrams related to EC-SERS effect show a peak 3.5 times more intense during the first cycle than in the second one (Fig. S4A). On the contrary, voltaRamangrams linked to EC-SOERS effect show the opposite effect (Fig. S4B). The changes observed in the CVs are very similar to those plotted for U molecule (Fig. S4C).

To simplify the understanding of our results, a summary of the aforementioned conclusions can be found in Figs. 2 and 3. Fig. 2A shows the CV and the voltaRamangrams under the experimental conditions in which the maximum EC-SOERS and EC-SERS intensities were registered for 5-FU. This means that in Fig. 2A it is plotted the signals in the cathodic region (from -0.10 to $-0.40 \mathrm{~V}$ ) for the first potential cycle, where the highest EC-SERS signal is registered, and the signals in the anodic region (from -0.10 to $+0.40 \mathrm{~V}$ ) for the second potential cycle, where the highest EC-SOERS signal is registered.

Several points should be noted about Fig. 2. First, two different Raman spectra have been obtained for the same molecule (5-FU) in one single experiment (Fig. 2B). The spectra differ in the number of observed bands, but the most representative Raman signals of 5-FU can be observed both at anodic and cathodic potentials. The differences can be related to the two different phenomena involved in the Raman enhancement during the experiment: EC-SERS and EC-SOERS. It is believed that EC-SOERS mechanism should involve some kind of electromagnetic enhancement (EM), since the analytical enhancement factors obtained with this method are too high to attribute them only to a chemical enhancement $[4,11,45]$. However, the different Raman spectra observed in EC-SERS and ECSOERS suggest that the structures responsible for this EM are different in EC-SERS and EC-SOERS. The shifting of several peaks, as well as the disappearance of many signals in EC-SOERS spectra, suggest that the interaction between the analyte and the metallic nanostructure responsible for each phenomenon should be substantially different.

This double fingerprint (EC-SERS and EC-SOERS spectrum) can be also observed for U molecule (Fig. 3) in a similar way as for 5FU molecule. When experiments shown in Figs. 2 and 3 are compared with a blank test, it can be observed that none of the as- 

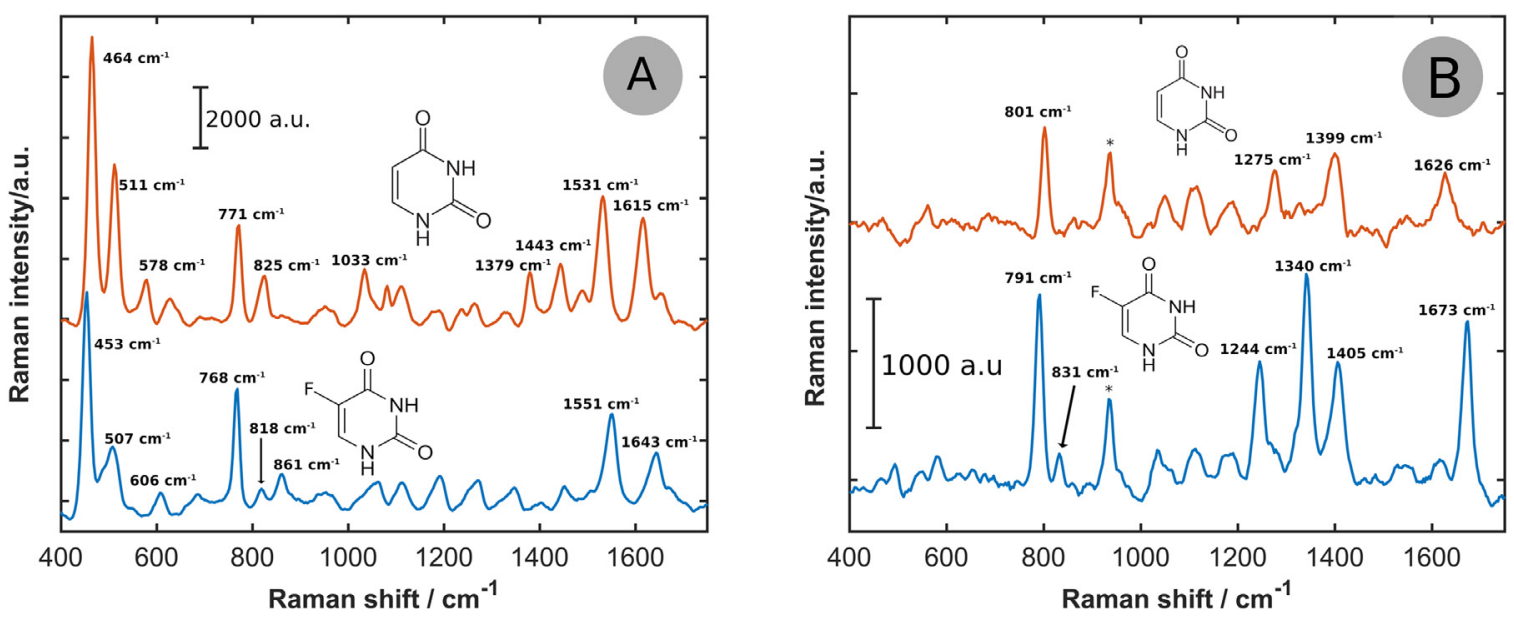

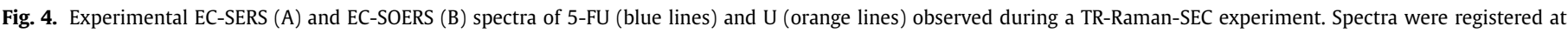

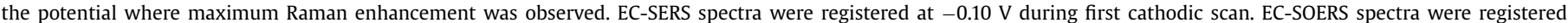

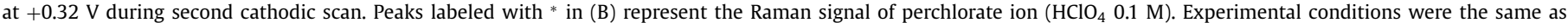
in Fig. 1. (For interpretation of the references to color in this figure legend, the reader is referred to the web version of this article.)

signed Raman bands of $\mathrm{U}$ or 5-FU are observed in the absence of these molecules (Fig. S5)

\subsection{Spectral differences}

Fig. 4A shows the EC-SERS spectra of 5-FU and U during a TRRaman-SEC experiment, under the same experimental conditions presented in Figs. 2 and 3. In this figure, it can be seen that the Raman spectra of the two molecules share many bands because of its great structural similarity. Nevertheless, some differences can be observed between the EC-SERS spectra of 5-FU and $U$ that can be analyzed as follows.

EC-SERS spectra of $U$ (Fig. 4A, orange line) is characterized by two strong bands at 464 and $511 \mathrm{~cm}^{-1}$. The assignation of these bands is not easy, to the best of our knowledge, these two bands have not been reported over silver substrates previously, at least no with the high intensity observed in our experiments [46-48].

These differences between our results and the reported SERS spectra of $U$ might be related to experimental conditions, such as $\mathrm{pH}$. Most authors have studied the SERS spectra of $U$ and uracil derivatives in alkaline or neutral media, where several deprotonated forms are present in solution. Under these conditions, adsorption processes of these ions can be expected [46,49]. Our work, however, is developed in acidic media $\left(\mathrm{pH}=1,0.1 \mathrm{M} \mathrm{HClO}_{4}\right)$, where $\mathrm{U}$ molecule is presented in protonated form, affecting to the interaction between the molecule and the surface. The dynamic generation of EC-SERS substrate is another crucial factor that can differentiate our spectra, since it has been observed that potential can play a huge role in $U$ angle of adsorption on silver surfaces [47].

Another difference that we can observed between our EC-SERS spectra and those reported in bibliography is a noticeable redshifting of the ring breathing band, which appears at $768 \mathrm{~cm}^{-1}$ for $U$ molecule in our experiment but has been usually reported around $800 \mathrm{~cm}^{-1}$ [46-48]. The shifting of this band has been usually related to the presence of various tautomeric forms of $U$, product of the deprotonation of $\mathrm{N}_{1}$ or $\mathrm{N}_{3}$ (Fig. 5) [46]. Usually, it has been proposed that $\mathrm{N}_{3}$ tautomer of $\mathrm{U}$ and 5-FU, rather than $\mathrm{N}_{1}$, is the main compound adsorbed on silver substrates [46-48,50], adsorbing via the negatively charged nitrogen. One of the marker bands used to characterize this interaction is the one at $800 \mathrm{~cm}^{-1}$ $[46,51]$. In our experiment, however, the shifting of this band, as well as the presence of a strong band at $1531 \mathrm{~cm}^{-1}$, suggest that the interaction between $U$ and silver is not taking place following the traditional mechanism during EC-SERS.

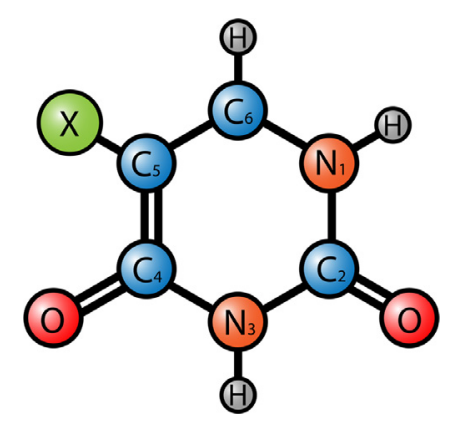

Fig. 5. Structure and atomic labeling of the studied analytes. $X=F$ for 5 -FU and $X=H$ for $U$.

The EC-SERS spectrum of 5-FU (Fig. 4A, blue line) can be analyzed in a similar way as the $U$ spectra. Again, the spectrum is dominated by two intense Raman bands at 453 and $507 \mathrm{~cm}^{-1}$, showing the first band a red-shifting $\left(\sim 15 \mathrm{~cm}^{-1}\right)$ respect to the same band for $U$. The ring breathing band, appearing at $768 \mathrm{~cm}^{-1}$, is also red-shifted $\left(>30 \mathrm{~cm}^{-1}\right)$ in comparison with those bands reported in SERS spectra of 5-FU on silver substrates in alkaline media [51]. At higher Raman shift, we find two relatively intense bands, at 1551 and $1643 \mathrm{~cm}^{-1}$, corresponding to the stretching mode of $C=C$ and $C=0$. These two bands suffer a blue-shifting (20$30 \mathrm{~cm}^{-1}$ ) with respect to the same bands for $U$.

As it was exposed, the observed EC-SERS spectra of $U$ and 5FU in our experiments seem to differ from those reported by other authors, both in the number and shifting of Raman bands. These facts suggest that both molecules, in acidic media, do not interact with silver surfaces in the same way as they do in alkaline media (via the anionic $\mathrm{N}_{3}$ tautomer).

The EC-SOERS spectra, however, seem much more similar to the reported SERS spectra of both, $U$ and 5-FU in alkaline media. EC-SOERS spectrum of $U$ (Fig. $4 \mathrm{~B}$, orange line) is dominated by a strong band at $801 \mathrm{~cm}^{-1}$, related with the in-plane deformation of aromatic ring. Another intense band can be observed at $1399 \mathrm{~cm}^{-1}$. Finally, it can be seen at $1626 \mathrm{~cm}^{-1}$ the band associated with the stretching of $\mathrm{C}=\mathrm{O}$. EC-SOERS spectrum of 5 -FU (Fig. $4 \mathrm{~B}$, blue line) is quite similar to the one observed for $U$, with the addition of some new bands at $831 \mathrm{~cm}^{-1}$, related with the out-of-plane $\mathrm{C}_{6}-\mathrm{H}$ distortion, and another intense band at $1340 \mathrm{~cm}^{-1} . \mathrm{C}=0$ stretching mode is present at $1673,40 \mathrm{~cm}^{-1}$ higher than the same band for $\mathrm{U}$. 
Table 1

Raman wavenumbers $\left(\mathrm{cm}^{-1}\right)$ of EC-SOERS and EC-SERS spectra of 5-FU and non-scaled, PBE0/(LANL2DZ,def2-SVP) wavenumbers of the structure I and

\begin{tabular}{|c|c|c|c|c|}
\hline Structure I & EC-SOERS signal & Structure II & EC-SERS signal & Assignment \\
\hline- & - & 400 & - & Scissoring $\mathrm{C}_{2}-\mathrm{O}$ and $\mathrm{C}_{4}-\mathrm{O}$ \\
\hline 408 & - & - & - & Out of plane bend of $\mathrm{C}_{6}-\mathrm{H}+$ out of plane bend of ring \\
\hline 451 & - & - & 453 & In plane bend $\mathrm{C}_{2}-\mathrm{O}$ and $\mathrm{C}_{4}-\mathrm{O}$ \\
\hline - & - & - & 507 & - \\
\hline 577 & - & 575 & - & Out of plane bending of $\mathrm{N}_{1}-\mathrm{H}$ \\
\hline- & - & - & 606 & - \\
\hline - & - & 675 & - & Out of plane bending of $\mathrm{N} 3 \mathrm{H}$ \\
\hline 800 & 791 & 763 & 768 & Ring breathing \\
\hline - & - & 763 & - & Out-of-plane bending of $\mathrm{N}_{1}-\mathrm{C}_{2}-\mathrm{N}_{3}-\mathrm{C}_{4}$ \\
\hline 845 & 831 & 827 & 818 & In-plane bending $\mathrm{C}_{6}-\mathrm{N}_{1}-\mathrm{C}_{2}-\mathrm{N}_{3}+$ stretching $\mathrm{C}-\mathrm{F}$ \\
\hline 884 & - & 879 & 861 & Out of plane bending $\mathrm{C}_{6}-\mathrm{H}$ \\
\hline 1171 & - & 1178 & - & Scissoring $\mathrm{C}_{6}-\mathrm{H}$ and $\mathrm{N}_{1}-\mathrm{H}$ \\
\hline 1302 & 1244 & 1305 & - & Stretching $\mathrm{C}-\mathrm{F}+$ asymmetric stretching of $\mathrm{N}-\mathrm{C}-\mathrm{N}$ \\
\hline 1384 & 1340 & 1357 & - & Stretching ring + symmetric rocking of $\mathrm{C}_{6}-\mathrm{H}$ and $\mathrm{N} 1-\mathrm{H}$ \\
\hline 1430 & 1405 & 1406 & - & Asymmetric stretching $\mathrm{N}_{1}-\mathrm{C}_{2}-\mathrm{N}_{3}+$ in-plane bending $\mathrm{N}_{1}-\mathrm{H}+$ stretching C-F \\
\hline 1536 & - & 1534 & 1551 & $\mathrm{C}_{4}-\mathrm{C}_{5}$ stretching + in-plane bend $\mathrm{N}_{1}-\mathrm{H}+$ asymmetric stretching of $\mathrm{C}_{6}-\mathrm{N}_{1}-\mathrm{C}_{2}$ \\
\hline 1675 & - & - & - & Asymmetric stretching of $\mathrm{C}_{2}-\mathrm{O}$ and $\mathrm{C}_{4}-\mathrm{O}$ \\
\hline 1747 & 1673 & - & - & Symmetric stretching $\mathrm{C}_{2}-\mathrm{O} \& \mathrm{C}_{4}-\mathrm{O}$ \\
\hline 1767 & - & 1743 & - & Stretching of $\mathrm{C}_{5}-\mathrm{C}_{6}+$ in-plane bend $\mathrm{C}_{6}-\mathrm{H}$ \\
\hline - & - & 1817 & 1643 & Stretching $\mathrm{C} 4-\mathrm{O}$ \\
\hline - & - & 1862 & & Stretching $\mathrm{C} 2-\mathrm{O}$ \\
\hline
\end{tabular}

It is noticeable the similarity of our EC-SOERS spectra of U and 5-FU with respect to the SERS spectra of these compounds reported by other authors. This fact suggests that the interaction between $U$ and 5-FU and the structure responsible for EC-SOERS could be similar to the interaction that takes place in SERS effect in alkaline media: adsorption of a deprotonated form of $U$ or 5-FU on silver structures via the negatively charged $\mathrm{N}$.

\subsection{DFT calculations}

To shed more light on the spectra observed during the spectroelectrochemistry experiment, we performed DFT calculations to evaluate the possible presence of various protonated forms of $U$ and 5-FU. Two different structures; structure I and II (Fig. S6), were optimized for each analyte as described above, resulting in a total of 4 simulated structures. All optimized structures can be found in supplementary materials. In all simulations, a cluster of $32 \mathrm{Ag}$ atoms was designed to emulate the surface of a silver nanostructure, and a molecule of $U$ or 5-FU was adsorbed on the (100) facet of the simulated cluster. In structure I, the molecule (U or 5-FU) is positioned perpendicular to the Ag surface, and in structure II, the molecule is positioned parallel to the surface. A remarkable difference between structure I and II is the protonation state of molecule: in structure I, the $\mathrm{N}_{3}$ atom of the adsorbed molecule has been deprotonated to ease the interaction with the silver substrate. However, in structure II, the molecule is in its neutral form and it is adsorbed on the surface horizontally.

Simulation of the Raman spectra of structure I and II of $U$ and 5-FU were performed, and its comparation with the EC-SOERS and EC-SERS for both analytes is represented in Tables 1 and 2. In these tables, non-scaled wavenumbers of optimized structures are shown.

In Table 1, it can be observed that a reasonable correlation exists between EC-SOERS signal and predicted signals for structure I of 5-FU. The displacements and number of bands predicted for structure I are similar to the experimental bands observed in ECSOERS. EC-SERS spectra of 5-FU, however, has more divergence between experimental and predicted signals. First, neither of the simulated Raman predicts the existence of two bands at 450-500 $\mathrm{cm}^{-1}$. Simulation of structure I reports a band at $451 \mathrm{~cm}^{-1}$ related with the in-plane bending (scissoring) of carbonyls. However, the band at $507 \mathrm{~cm}^{-1}$ remains unexplained.
One interesting band is the breathing mode of ring, located around $750-800 \mathrm{~cm}^{-1}$. For structure I of 5-FU, this band is calculated at $800 \mathrm{~cm}^{-1}$, and for structure II of same analyte it is calculated at $763 \mathrm{~cm}^{-1}$ (Table 1). The same trend is observed for $U$, in Table 2 . This variability in the prediction should be related with the protonation estate of the molecule, since the vibrational modes associated to this band do not involve the vibration of silver atoms; it only involves the vibration of the atoms of 5-FU or $\mathrm{U}$. This divergence in the calculated frequencies can be expected, since the resonant forms of $\mathrm{N}_{3}$ deprotonated molecule (structure I) would increase bond order among the atoms conforming the ring, thus increasing the energy associated to the stretching mode of these bonds. Similar conclusions can be extracted from the comparison of the in-plane bending of ring, located at $\sim 830 \mathrm{~cm}^{-1}$

This variability in the in-plane deformation of ring modes is also present in the experimental EC-SOERS and EC-SERS spectra. We propose that the molecular species involved in both phenomena should be in different protonated forms, with the deprotonated form of $U$ and 5-FU probably being the one involved in EC-SOERS phenomenon.

Although it has been stablished that EC-SOERS is a phenomenon that takes place in acidic media [6,11], at $\mathrm{pH}$ much lower than the $\mathrm{pK}_{\mathrm{a}}$ of 5 -FU or $\mathrm{U}$ [51], it is possible that the applied potential during silver oxidation, potentials at which EC-SOERS is observed, could be helping to break the bond between $\mathrm{N}-\mathrm{H}$, since it has been reported that anodic potential can modify the $\mathrm{pK}_{\mathrm{a}}$ of carboxylic acids on silver substrates [52]. Moreover, it has been proposed that potential-assisted deprotonation of pyridinic nitrogen of adenine can take place on gold surfaces [53].

One of the main differences between EC-SOERS and EC-SERS spectra of both analytes is the presence of $\mathrm{C}=\mathrm{C}$ stretching band $\left(\sim 1540 \mathrm{~cm}^{-1}\right)$, which is notoriously intense for EC-SERS spectra but is not even present in EC-SOERS. Our calculations suggest that in the two proposed structures this band should have a moderately intense Raman activity, so this remarkable difference between spectra could be related to the selection rules involved in each phenomenon. Further theoretical and experimental efforts should be done in order to find a reason for these changes in the number of Raman bands between spectra.

Finally, we found a noticeable shifting in the carbonyl stretching bands $\left(\sim 1650 \mathrm{~cm}^{-1}\right)$. For EC-SOERS spectra, this band appears at 
Table 2

Raman wavenumbers $\left(\mathrm{cm}^{-1}\right)$ of EC-SOERS and EC-SERS spectra of $U$ and non-scaled, PBE0/(LANL2DZ,def2-SVP) wavenumbers of the structure I and II.

\begin{tabular}{|c|c|c|c|c|}
\hline Structure I & EC-SOERS signal & Structure II & EC-SERS signal & Assignment \\
\hline- & - & 407 & - & Out of-plane bend of $\mathrm{C} 5-\mathrm{H}$ \\
\hline 454 & - & - & 464 & in-plane symmetric bend of $\mathrm{C}-\mathrm{O}$ \\
\hline- & - & - & 511 & - \\
\hline 561 & - & 571 & 578 & in-plane ring bend \\
\hline 595 & - & - & - & in-plane ring stretching \\
\hline 603 & - & 603 & 627 & out-of-plane bend of $\mathrm{N} 1-\mathrm{H}$ \\
\hline 736 & - & 728 & - & out-of-plane bend of $\mathrm{C} 5-\mathrm{H}+$ out-of-plane bend of ring \\
\hline 790 & - & 774 & - & out of plane ring deformation \\
\hline 815 & 801 & 795 & 771 & Ring breathing \\
\hline 822 & - & 811 & 825 & out-of-plane bend of $\mathrm{C} 6-\mathrm{H}, \mathrm{C} 5-\mathrm{H}$ and $\mathrm{C} 4$ \\
\hline- & - & - & 1033 & - \\
\hline 1209 & 1106 & 1214 & 1112 & scissoring of $\mathrm{H}-\mathrm{N} 1-\mathrm{C} 6-\mathrm{H}$ and $\mathrm{H}-\mathrm{C} 6-\mathrm{C} 5-\mathrm{H}$ \\
\hline- & - & 1253 & - & rocking of $\mathrm{N} 3-\mathrm{H}, \mathrm{N} 1-\mathrm{H}$ and $\mathrm{C} 5-\mathrm{H}+$ asymmetric stretching of $\mathrm{C} 2-\mathrm{N} 3-\mathrm{C} 4$ \\
\hline- & 1281 & - & - & - \\
\hline 1392 & - & 1393 & 1379 & rocking of $\mathrm{N} 1-\mathrm{H}, \mathrm{C} 5-\mathrm{H}$ and $\mathrm{C} 6-\mathrm{H}$ \\
\hline- & - & 1420 & - & In-plane bend of $\mathrm{N} 3-\mathrm{H}, \mathrm{C} 5-\mathrm{H}$ and $\mathrm{C} 6-\mathrm{H}$ \\
\hline 1404 & 1399 & 1437 & 1443 & in-plane bend of $\mathrm{N} 1-\mathrm{H}+$ symmetric stretching of $\mathrm{C} 2-\mathrm{N} 3-\mathrm{C} 4$ \\
\hline 1535 & - & 1525 & 1531 & C4-C5 stretching + in-plane bend of $\mathrm{N} 1-\mathrm{H}+$ stretching of C6-N1 \\
\hline 1653 & - & - & - & asymmetric stretching of $\mathrm{C}-\mathrm{O}+$ in-plane bend of $\mathrm{N} 1-\mathrm{H}$ \\
\hline 1722 & - & 1690 & - & stretching $\mathrm{C} 5-\mathrm{C} 6+$ rocking $\mathrm{H}-\mathrm{C} 5-\mathrm{N} 1-\mathrm{H}$ \\
\hline 1746 & 1630 & - & - & symmetric stretching $\mathrm{C}-\mathrm{O}+$ in-plane bend of $\mathrm{N} 1-\mathrm{H}$ \\
\hline- & - & 1783 & 1615 & stretching $\mathrm{C} 4=0$ \\
\hline- & - & 1826 & & Stretching $\mathrm{C} 2=0$ \\
\hline
\end{tabular}

higher Raman shift than the same bands in EC-SERS spectra, which suggests that different interaction between oxygen and silver substrate is taking place in these phenomena.

As has been experimentally demonstrated and theoretically discussed, EC-SERS and EC-SOERS spectra provide a different fingerprint for the same molecule. Electrochemistry can be used for the generation of the two Raman signal amplification phenomena. Therefore, a double fingerprint can be obtained for the same molecule, with TR-Raman-SEC being a double detection technique in a single experiment. This strategy could be used for analysis, providing more reliable results for the identification of molecules in samples. In addition, DFT simulations provides new insights about EC-SOERS.

\section{Conclusions}

TR-Raman-SEC has been used to obtain, for the first time ever, two characteristic spectroscopic fingerprints of the same analyte in a single experiment, as has been demonstrated using $U$ and 5-FU, two analytes of great relevance in biochemistry and pharmacology. For this purpose, two different Raman enhancement phenomena have been electrochemically generated, EC-SERS and EC-SOERS, being each phenomenon observable during different stages of the Raman spectroelectrochemistry experiment. These two spectroscopic fingerprints show differences between them, but correspond unequivocally to the same analyte.

DFT calculations has been performed to elucidate the interaction between substrate and analyte. It has been proposed the presence of potential-aided deprotonated species during EC-SOERS phenomenon.

We think that this methodology has a great potential in analysis, since the use of two Raman enhancement phenomena, each one providing a unique fingerprint of analyte, can increase the power of Raman spectroscopy to resolve complex interferences between various analytes. Further theoretical studies could provide more information about the processes taking place during EC-SOERS and EC-SERS, increasing the analytical potential of this methodology.

\section{Declaration of Competing Interest}

The authors declare that they have no known competing financial interests or personal relationships that could have appeared to influence the work reported in this paper.

\section{Credit authorship contribution statement}

Martin Perez-Estebanez: Data curation, Conceptualization, Visualization, Formal analysis, Writing - original draft, Writing - review \& editing. William Cheuquepan: Data curation, Conceptualization, Visualization, Formal analysis, Writing - original draft, Writing - review \& editing. Jose V. Cuevas-Vicario: Writing - original draft, Writing - review \& editing. Sheila Hernandez: Conceptualization, Visualization, Formal analysis, Writing - original draft, Writing - review \& editing. Aranzazu Heras: Conceptualization, Visualization, Formal analysis, Writing - original draft, Writing review \& editing. Alvaro Colina: Conceptualization, Visualization, Formal analysis, Writing - original draft, Writing - review \& editing.

\section{Acknowledgments}

Authors acknowledge the financial support from Ministerio de Economía, y Competitividad (Grant CTQ2017-83935-RAEI/FEDERUE), Junta de Castilla y León (Grant BU297P18, Grant BU087G19, and Grant BU263P18) and Ministerio de Ciencia, Innovación y Universidades (Grant RED2018-102412-T and Grant PID2019-111215RB-I00). W. Ch. thanks JCyL for his postdoctoral fellowship (Grant BU297P18). S.H. thanks JCyL and European Social Fund for her predoctoral fellowship. M.P-E. thanks JCyL, the European Social Fund and the Youth Employment initiative and JCyL and European Social Fund for his predoctoral fellowship. This research has made use of the high-performance computing resources of the Castilla y León Supercomputing Center (SCAYLE, https://www.scayle.es), financed by FEDER (Fondo Europeo de Desarrollo Regional). Jorge Gonzalez is acknowledged for his help in the laboratory whose contract was founded by JCyL, the European Social Fund and the Youth Employment Initiative. 


\section{Supplementary materials}

Supplementary material associated with this article can be found, in the online version, at doi:10.1016/j.electacta.2021.138615.

\section{References}

[1] M. Fan, G.F.S. Andrade, A.G. Brolo , A review on the fabrication of substrates for surface enhanced Raman spectroscopy and their applications in analytical chemistry, Anal. Chim. Acta 693 (2011) 7-25, doi:10.1016/j.aca.2011.03.002.

[2] M. Fleischmann, P.J. Hendra, A.J. McQuillan , Raman spectra of pyridine adsorbed at a silver electrode, Chem. Phys. Lett. 26 (1974) 163-166, doi:10.1016/ 0009-2614(74)85388-1.

[3] S. Schlücker , Surface-enhanced Raman spectroscopy: concepts and chemical applications, Angew. Chem. Int. Ed. 53 (2014) 4756-4795, doi:10.1002/anie. 201205748.

[4] J. Langer, D. Jimenez de Aberasturi, J. Aizpurua, R.A. Alvarez-Puebla, B. Auguié, J.J. Baumberg, G.C. Bazan, S.E.J. Bell, A. Boisen, A.G. Brolo, J. Choo, D. Cialla-May, V. Deckert, L. Fabris, K. Faulds, F.J. García de Abajo, R. Goodacre, D. Graham, A.J. Haes, C.L. Haynes, C. Huck, T. Itoh, M. Käll, J. Kneipp, N.A. Kotov, H. Kuang, E.C. Le Ru, H.K. Lee, J.F. Li, X.Y. Ling, S.A. Maier, T. Mayerhöfer, M. Moskovits, K. Murakoshi, J.M. Nam, S. Nie, Y. Ozaki, I. Pastoriza-Santos, J. Perez-Juste, J. Popp, A. Pucci, S. Reich, B. Ren, G.C. Schatz, T. Shegai, S. Schlücker, L.L. Tay, K.G. Thomas, Z.Q. Tian, R.P. Van Duyne, T. Vo-Dinh, Y. Wang, K.A. Willets, C. Xu, H. Xu, Y. Xu, Y.S. Yamamoto, B. Zhao, L.M. Liz-Marzán , Present and future of surface-enhanced Raman scattering, ACS Nano 14 (2020) 28-117, doi:10.1021/acsnano.9b04224.

[5] B. Sharma, R.R. Frontiera, A.I. Henry, E. Ringe, R.P. Van Duyne , SERS: materials, applications, and the future, Mater. Today 15 (2012) 16-25, doi:10.1016/ S1369-7021(12)70017-2.

[6] J.V. Perales-Rondon, S. Hernandez, D. Martin-Yerga, P. Fanjul-Bolado, A. Heras, A. Colina , Electrochemical surface oxidation enhanced Raman scattering, Electrochim. Acta 282 (2018) 377-383, doi:10.1016/j.electacta.2018.06.079.

[7] L. Bao, S.M. Mahurin, S. Dai , Controlled layer-by-layer formation of ultrathin $\mathrm{TiO}_{2}$ on silver island films via a surface sol-gel method for surfaceenhanced Raman scattering measurement, Anal. Chem. 76 (2004) 4531-4536, doi:10.1021/ac049668c.

[8] E.V. Formo, S.M. Mahurin, S. Dai, Robust SERS substrates generated by coupling a bottom-up approach and atomic layer deposition, ACS Appl. Mater. Interfaces 2 (2010) 1987-1991, doi:10.1021/am100272h.

[9] S. Hernandez, J.V. Perales-Rondon, A. Heras, A. Colina , Determination of uric acid in synthetic urine by using electrochemical surface oxidation enhanced Raman scattering, Anal. Chim. Acta 1085 (2019) 61-67, doi:10.1016/j.aca.2019. 07.057.

[10] S. Hernandez, J.V. Perales-Rondon, A. Heras, A. Colina , Electrochemical SERS and SOERS in a single experiment: a new methodology for quantitative analysis, Electrochim. Acta 334 (2020) 135561, doi:10.1016/j.electacta.2019.135561.

[11] M. Perez-Estebanez, S. Hernandez, J.V. Perales-Rondon, E. Gomez, A. Heras, A. Colina , Chemical selectivity in electrochemical surface oxidation enhanced Raman scattering, Electrochim. Acta 353 (2020) 136560, doi:10.1016/j.electacta. 2020.136560.

[12] R. Li, H. Lv, X. Zhang, P. Liu, L. Chen, J. Cheng, B. Zhao, Vibrational spectroscopy and density functional theory study of 4-mercaptobenzoic acid, Spectrochim. Acta Part A Mol. Biomol. Spectrosc. 148 (2015) 369-374, doi:10.1016/j.saa.2015. 03.132.

[13] Y. Chen, J. Yang, Z. Li, R. Li, W. Ruan, Z. Zhuang, B. Zhao , Experimental and density functional theory study of Raman and SERS spectra of 5-amino-2mercaptobenzimidazole, Spectrochim. Acta Part A Mol. Biomol. Spectrosc. 153 (2016) 344-348, doi:10.1016/j.saa.2015.08.039.

[14] R. Li, Z. Mao, L. Chen, H. Lv, J. Cheng, B. Zhao, Vibrational spectroscopy and density functional theory study of 3-[4,5-dimethyl-2-thiazolyl]-2,5-diphenyl$2 \mathrm{H}$-tetrazolium bromide, Spectrochim. Acta Part A Mol. Biomol. Spectrosc. 135 (2015) 1-6, doi:10.1016/j.saa.2014.06.084.

[15] M.L. Xu, Y. Gao, X.X. Han, B. Zhao , Detection of pesticide residues in food using surface-enhanced Raman spectroscopy: a review, J. Agric. Food Chem. 65 (2017) 6719-6726, doi:10.1021/acs.jafc.7b02504

[16] D. Ramesh, B.G. Vijayakumar, T. Kannan , Therapeutic potential of uracil and its derivatives in countering pathogenic and physiological disorders, Eur. J. Med. Chem. 207 (2020) 112801, doi:10.1016/j.ejmech.2020.112801.

[17] D. Zhu, Y. Wei, J. Yin, D. Liu, E.L. Ang, H. Zhao, Y. Zhang , A pathway for degradation of uracil to acetyl coenzyme A in bacillus megaterium, Appl. Environ. Microbiol. 86 (2020) 1-10, doi:10.1128/AEM.02837-19.

[18] H.E. Krokan, F. Drabløs, G. Slupphaug , Uracil in DNA - occurrence, consequences and repair, Oncogene 21 (2002) 8935-8948, doi:10.1038/sj.onc. 1205996.

[19] D.B. Longley, D.P. Harkin, P.G. Johnston , 5-Fluorouracil: mechanisms of action and clinical strategies, Nat. Rev. Cancer 3 (2003) 330-338, doi:10.1038/nrc1074.

[20] S. Christensen, B. Van der Roest, N. Besselink, R. Janssen, S. Boymans, J.W.M. Martens, M.L. Yaspo, P. Priestley, E. Kuijk, E. Cuppen, A. Van Hoeck , 5-Fluorouracil treatment induces characteristic T\&GT;G mutations in human cancer, Nat. Commun. 10 (2019) 4571, doi:10.1038/s41467-019-12594-8.

[21] Y. Ai, O.N. Obianom, M. Kuser, Y. Li, Y. Shu, F. Xue , Enhanced tumor selectivity of 5-fluorouracil using a reactive oxygen species-activated prodrug approach, ACS Med. Chem. Lett. 10 (2019) 127-131, doi:10.1021/acsmedchemlett. 8 b00539.
[22] S.T.J. Kalpana Ghoshal, Specific Inhibition of pre-ribosomal RNA processing in extracts from the lymphosarcoma cells treated with 5-fluorouracil, Cancer Res. 54 (1994) 632-636, https://pubmed.ncbi.nlm.nih.gov/8306322/.

[23] R. Kanamaru, H. Kakuta, T. Sato, C. Ishioka, A. Wakui , The inhibitory effects of 5-fluorouracil on the metabolism of preribosomal and ribosomal RNA in L-1210 cells in vitro, Cancer Chemother. Pharmacol. 17 (1986) 43-46, doi:10. 1007/BF00299864.

[24] B. Büchel, P. Rhyn, S. Schürch, C. Bühr, U. Amstutz, C.R. Largiadèr , LC-MS/MS method for simultaneous analysis of uracil, 5,6-dihydrouracil, 5-fluorouracil and 5-fluoro-5,6-dihydrouracil in human plasma for therapeutic drug monitoring and toxicity prediction in cancer patients, Biomed. Chromatogr. 27 (2013) 7-16, doi:10.1002/bmc.2741.

[25] F.M. Zahed, B. Hatamluyi, F. Lorestani, Z. Es'haghi , Silver nanoparticles decorated polyaniline nanocomposite based electrochemical sensor for the determination of anticancer drug 5-fluorouracil, J. Pharm. Biomed. Anal. 161 (2018) 12-19, doi:10.1016/j.jpba.2018.08.004.

[26] M. Satyanarayana, K.Y. Goud, K.K. Reddy, K.V. Gobi , Biopolymer stabilized nanogold particles on carbon nanotube support as sensing platform for electrochemical detection of 5-fluorouracil in-vitro, Electrochim. Acta 178 (2015) 608-616, doi:10.1016/j.electacta.2015.08.036

[27] M.S. Khot, S.L. Bhattar, G.B. Kolekar, S.R. Patil , Spectrofluorimetric determination of 5-fluorouracil by fluorescence quenching of 9-anthracenecarboxylic acid, Spectrochim. Acta Part A Mol. Biomol. Spectrosc. 77 (2010) 82-86, doi:10. 1016/j.saa.2010.04.029.

[28] D. Zhu, Y. Chen, L. Jiang, J. Geng, J. Zhang, J.J. Zhu , Manganese-doped ZnSe quantum dots as a probe for time-resolved fluorescence detection of 5fluorouracil, Anal. Chem. 83 (2011) 9076-9081, doi:10.1021/ac202101u.

[29] K.C. Bantz, A.F. Meyer, N.J. Wittenberg, H. Im, Ö. Kurtuluş, S.H. Lee, N.C. Lindquist, S.H. Oh, C.L. Haynes, Recent progress in SERS biosensing, Phys. Chem. Chem. Phys. 13 (2011) 11551, doi:10.1039/c0cp01841d.

[30] H.F. Nargis, H. Nawaz, H.N. Bhatti, K. Jilani, M. Saleem , Comparison of surface enhanced Raman spectroscopy and Raman spectroscopy for the detection of breast cancer based on serum samples, Spectrochim. Acta Part A Mol. Biomol. Spectrosc. 246 (2021) 119034, doi:10.1016/j.saa.2020.119034.

[31] H.G. Lee, W. Choi, S.Y. Yang, D.H. Kim, S.G. Park, M.Y. Lee, H.S. Jung , PCRcoupled paper-based surface-enhanced Raman scattering (SERS) sensor for rapid and sensitive detection of respiratory bacterial DNA, Sens. Actuators B Chem. 326 (2021) 128802, doi:10.1016/j.snb.2020.128802.

[32] S. Ganesh, K. Venkatakrishnan, B. Tan , Quantum scale organic semiconductors for SERS detection of DNA methylation and gene expression, Nat. Commun. 11 (2020) 1135, doi:10.1038/s41467-020-14774-3.

[33] J. Zeng, M. Dong, B. Zhu, D. Chen, Y. Li , A new method towards the detection of DNA mutation by surface-enhanced Raman spectroscopy, Talanta 223 (2021) 121746, doi:10.1016/j.talanta.2020.121746.

[34] M.B. Mamián-López, M.L.A. Temperini , On the cooperativity effect in Watson and Crick and Wobble pairs for a halouracil series and its potential quantitative application studied through surface-enhanced Raman spectroscopy, Anal. Chem. 90 (2018) 14165-14172, doi:10.1021/acs.analchem.8b02188.

[35] C. Adamo, V. Barone, Toward reliable density functional methods without adjustable parameters: the PBE0 model, J. Chem. Phys. 110 (1999) 6158-6170, doi: $10.1063 / 1.478522$

[36] S. Grimme, J. Antony, S. Ehrlich, H. Krieg , A consistent and accurate ab initio parametrization of density functional dispersion correction (DFT-D) for the 94 elements H-Pu, J. Chem. Phys. 132 (2010) 154104, doi:10.1063/1.3382344.

[37] S. Grimme, S. Ehrlich, L. Goerigk , Effect of the damping function in dispersion corrected density functional theory, J. Comput. Chem. 32 (2011) 1456-1465, doi: $10.1002 /$ jcc. 21759.

[38] F. Weigend, R. Ahlrichs, Balanced basis sets of split valence, triple zeta valence and quadruple zeta valence quality for $\mathrm{H}$ to $\mathrm{Rn}$ : design and assessment of accuracy, Phys. Chem. Chem. Phys. 7 (2005) 3297, doi:10.1039/b508541a.

[39] P.J. Hay, W.R. Wadt, Ab initio effective core potentials for molecular calculations. Potentials for $\mathrm{K}$ to $\mathrm{Au}$ including the outermost core orbitals, J. Chem. Phys. 82 (1985) 299-310, doi:10.1063/1.448975.

[40] F. Neese, Software update: the ORCA program system, version 4.0, WIREs Comput. Mol. Sci. 8 (2018), doi:10.1002/wcms.1327.

[41] F. Neese, The ORCA program system, WIREs Comput. Mol. Sci. 2 (2012) 73-78, doi: $10.1002 /$ wcms.81.

[42] J.V. Perales-Rondon, S. Hernandez, D. Martin-Yerga, P. Fanjul-Bolado, A. Heras, A. Colina, Electrochemical surface oxidation enhanced Raman scattering, Electrochim. Acta 282 (2018) 377-383, doi:10.1016/j.electacta.2018.06.079.

[43] J.V. Perales-Rondon, S. Hernandez, A. Heras, A. Colina, Effect of chloride and $\mathrm{pH}$ on the electrochemical surface oxidation enhanced Raman scattering, Appl. Surf. Sci. 473 (2019) 366-372, doi:10.1016/j.apsusc.2018.12.148.

[44] D.-.Y. Wu, J.F. Li, B. Ren, Z.Q. Tian, Electrochemical surface-enhanced Raman spectroscopy of nanostructures, Chem. Soc. Rev. 37 (2008) 1025-1041, doi:10. 1039/b707872m

[45] B. Sharma, R.R. Frontiera, A. Henry, E. Ringe, R.P. Van Duyne, SERS: materials, applications, and the future, Mater. Today 15 (2012) 16-25, doi:10.1016/ S1369-7021(12)70017-2.

[46] B. Giese, D. McNaughton, Surface-enhanced Raman spectroscopic study of uracil. the influence of the surface substrate, surface potential, and $\mathrm{pH}$, J. Phys. Chem. B 106 (2002) 1461-1470, doi:10.1021/jp011986h.

[47] Z.B. Lin, B.G. Xie, J.H. Tian, Y.A. Tang, J.J. Sun, G.N. Chen, B. Ren, B.W. Mao Z.Q. Tian, Potential-dependent adsorption of uracil on a silver electrode in alkaline solutions, J. Electroanal. Chem. 636 (2009) 74-79, doi:10.1016/j. jelechem.2009.09.014. 
[48] K.H. Cho, J. Choo, S.W. Joo, Surface-enhanced Raman scattering and density functional theory calculation of uracil on gold and silver nanoparticle surfaces, Spectrochim. Acta Part A Mol. Biomol. Spectrosc. 61 (2005) 1141-1145, doi:10. 1016/j.saa.2004.06.032.

[49] M. Sardo, C. Ruano, J.L. Castro, I. López-Tocón, J. Soto, P. Ribeiro-Claro, J.C. Otero, Surface-enhanced Raman scattering of 5-fluorouracil adsorbed on silver nanostructures, Phys. Chem. Chem. Phys. 11 (2009) 7437, doi:10.1039/ b903823j.

[50] I. Pavel, S. Cota, S. Cînta-Pînzaru, W. Kiefer, Raman, surface enhanced Raman spectroscopy, and dft calculations: a powerful approach for the identification and characterization of 5-fluorouracil anticarcinogenic drug species, J. Phys. Chem. A 109 (2005) 9945-9952, doi:10.1021/jp053626q.
[51] M. Sardo, C. Ruano, J.L. Castro, I. López-Tocón, J. Soto, P. Ribeiro-Claro, J.C. Otero, Surface-enhanced Raman scattering of 5-fluorouracil adsorbed on silver nanostructures, Phys. Chem. Chem. Phys. 11 (2009) 7437, doi:10.1039/ b903823j.

[52] C. Ma, J.M. Harris, Surface-enhanced Raman spectroscopy investigation of the potential-dependent acid-base chemistry of silver-immobilized 2mercaptobenzoic acid, Langmuir 27 (2011) 3527-3533, doi:10.1021/la1044859.

[53] N. Martín Sabanés, T. Ohto, D. Andrienko, Y. Nagata, K. F. Domke , Electrochemical TERS Elucidates Potential-Induced Molecular Reorientation of Adenine/Au(111), Angew. Chemie - Int. Ed. 56 (2017) 9796-9801, doi:10.1002/anie. 201704460. 